\title{
Khiops: A Statistical Discretization Method of Continuous Attributes
}

\author{
MARC BOULLE \\ France Telecom R\&D, 2, Avenue Pierre Marzin, 22300 Lannion, France \\ Editor: Peter Flach
}

marc.boulle@francetelecom.com

\begin{abstract}
In supervised machine learning, some algorithms are restricted to discrete data and have to discretize continuous attributes. Many discretization methods, based on statistical criteria, information content, or other specialized criteria, have been studied in the past. In this paper, we propose the discretization method Khiops, ${ }^{1}$ based on the chi-square statistic. In contrast with related methods ChiMerge and ChiSplit, this method optimizes the chisquare criterion in a global manner on the whole discretization domain and does not require any stopping criterion. A theoretical study followed by experiments demonstrates the robustness and the good predictive performance of the method.
\end{abstract}

Keywords: data mining, machine learning, discretization, data analysis

\section{Introduction}

Discretization of continuous attributes is a problem that has been studied extensively in the past (Catlett, 1991; Holte, 1993; Dougherty, Kohavi, \& Sahami, 1995; Zighed \& Rakotomalala, 2000). Many induction algorithms rely on discrete attributes, and need to discretize continuous attributes, i.e. to slice their domain into a finite number of intervals. For example, decision tree algorithms exploit a discretization method to handle continuous attributes. C4.5 (Quinlan, 1993) uses the information gain based on Shannon entropy. CART (Breiman, 1984) applies the Gini criterion (a measure of the impurity of the intervals). CHAID (Kass, 1980) relies on a discretization method close to ChiMerge (Kerber, 1991). SIPINA takes advantage of the Fusinter criterion (Zighed, 1998) based on measures of uncertainty that are sensitive to sample size. While most discretization methods are employed as a preprocessing step to an induction algorithm, there are still other approaches, similar to the wrapper approach involved in the feature selection field. For example, Bertelsen and Martinez (1994) and Burdsall and Giraud-Carrier (1997) propose approaches that allow to refine the discretization of the continuous explanatory attributes by taking feedback from an induction algorithm.

Most discretization methods are divided into top-down and bottom-up methods. Topdown methods start from the initial interval and recursively split it into smaller intervals. Bottom-up methods start from the set of single value intervals and iteratively merge neighboring intervals. Some of these methods require user parameters to modify the behavior of the discretization criterion or to set up a threshold for the stopping rule. In the discretization problem, a compromise must be found between information quality (homogeneous intervals 
in regard to the attribute to predict) and statistical quality (sufficient sample size in every interval to ensure generalization). The chi-square-based criteria focus on the statistical point of view whereas the entropy-based criteria focus on the information point of view. Other criteria (such as Gini or Fusinter criterion) try to find a trade off between information and statistical properties. The Minimum Description Length (MDL) criterion (Fayyad, 1992) is an original approach that attempts to minimize the total quantity of information both contained in the model and in the exceptions to the model.

We present a new discretization method named Khiops. This is a bottom-up method based on the global optimization of chi-square. The most similar existing methods are the topdown and bottom-up methods using the chi-square criterion in a local manner. The top-down method based on chi-square is ChiSplit (Bertier \& Bouroche, 1981). It searches for the best split of an interval, by maximizing the chi-square criterion applied to the two sub-intervals adjacent to the splitting point: the interval is split if both sub-intervals substantially differ statistically. The ChiSplit stopping rule is based on a user-defined chi-square threshold to reject the split if the two sub-intervals are too similar. The bottom-up method based on chi-square is ChiMerge (Kerber, 1991). It searches for the best merge of adjacent intervals by minimizing the chi-square criterion applied locally to two adjacent intervals: they are merged if they are statistically similar. The stopping rule is based on a user-defined chisquare threshold to reject the merge if the two adjacent intervals are insufficiently similar.

The Khiops method proposed in this paper starts the discretization from the elementary single value intervals. It evaluates all merges between adjacent intervals and selects the best one according to the chi-square criterion applied to the whole set of intervals. The stopping rule is based on the confidence level computed with the chi-square statistic. The method automatically stops merging intervals as soon as the confidence level, related to the chisquare test of independence between the discretized attribute and the class attribute, does not decrease anymore. The Khiops method optimizes a global criterion that evaluates the partition of the domain into intervals, as opposed to a local criterion applied to two neighboring intervals as in ChiSplit or ChiMerge. The absence of user parameters makes the method very convenient to use and allows to automatically obtain high-quality discretizations. We will demonstrate that in spite of this global approach, the Khiops approach can be implemented such that its run-time is super-linear in the sample size. This computational complexity is the same as that of the optimized version of the ChiMerge algorithm. We will compare the Khiops method with other discretization methods by means of experiments on standard benchmarks.

The remainder of the paper is organized as follows. Section 2 presents the Khiops algorithm and its main properties. Section 3 compares the Khiops method with the related ChiMerge and ChiSplit methods from a theoretical point of view. Section 4 proceeds with an extensive experimental evaluation.

\section{The Khiops discretization method}

In this section, we first recall the principles of the chi-square test and then present the Khiops algorithm. Next, we focus on the computational complexity of the algorithm, and finally, we discuss some practical issues. 
Table 1. Contingency table used to compute the chi-square value.

\begin{tabular}{|c|c|c|c|c|c|c|}
\hline$n_{i j}:$ & $\begin{array}{l}\text { Observed frequency for } i \text { th explanatory value } \\
\text { and } j \text { th class value }\end{array}$ & a & $\begin{array}{c}A \\
n_{11}\end{array}$ & $\begin{array}{c}B \\
n_{12}\end{array}$ & $\begin{array}{c}C \\
n_{13}\end{array}$ & $\begin{array}{c}\text { Total } \\
n_{1}\end{array}$ \\
\hline$n_{i .}:$ & Total observed frequency for $i$ th explanatory value & $\mathrm{b}$ & $n_{21}$ & $n_{22}$ & $n_{23}$ & $n_{2}$ \\
\hline$n_{. j}:$ & Total observed frequency for $j$ th class value & $\mathrm{c}$ & $n_{31}$ & $n_{32}$ & $n_{33}$ & $n_{3}$ \\
\hline$N:$ & Total observed frequency & d & $n_{41}$ & $n_{42}$ & $n_{43}$ & $n_{4}$ \\
\hline$I:$ & Number of explanatory attribute values & $\mathrm{e}$ & $n_{51}$ & $n_{52}$ & $n_{53}$ & $n_{5}$ \\
\hline$J:$ & Number of class values & Total & $n_{.1}$ & $n_{.2}$ & $n_{.3}$ & $N$ \\
\hline
\end{tabular}

\subsection{The chi-square test: Principles and notations}

Let us consider an explanatory attribute (a feature) and a class attribute, and determine whether they are independent. First, all instances are summarized in a contingency table, where the instances are counted for each value pair of explanatory and class attributes. The chi-square value is computed from the contingency table.

Let $e_{i j}=n_{i .} * n_{. j} / N . e_{i j}$ stands for the expected frequency for cell $(i, j)$ if the explanatory and class attributes are independent.

The chi-square value is a measure on the whole contingency table of the difference between observed frequencies and expected frequencies. It can be interpreted as a distance to the hypothesis of independence between attributes.

$$
\text { Chi2 }=\sum_{i} \sum_{j} \frac{\left(n_{i j}-e_{i j}\right)^{2}}{e_{i j}}
$$

If the hypothesis of independence is true, the chi-square value is distributed as a chi-square statistic with $(I-1) *(J-1)$ degrees of freedom. This is the basis for a statistical test that allows rejecting the hypothesis of independence. The confidence level is the probability of the hypothesis of independence. For example, in a contingency table of size $5 * 3$ (which corresponds to a chi-square statistic with 8 degrees of freedom), the confidence level associated to a chi-square value of 20 is about $1 \%$. This means that when the explanatory and the class attributes are independent, the probability of observing a chi-square value greater than 20 is less than $1 \%$. The higher the chi-square value is, the smaller is the confidence level.

\subsection{Algorithm}

The chi-square value depends both on the local observed frequencies in each individual row and on the global observed frequencies in the whole contingency table. This is a good candidate criterion for a discretization method. The chi-square statistic is parameterized by the number of explanatory values (related to the degrees of freedom). In order to compare two discretizations with different interval numbers, we will use the confidence level instead of the chi-square value.

The principle of the Khiops algorithm is to minimize the confidence level between the discretized explanatory attribute and the class attribute by means of the chi-square statistic. 
The chi-square value is not reliable to test the hypothesis of independence if the expected frequency in any cell of the contingency table is less than some minimum value. This is equivalent to a minimum frequency constraint for each row of the contingency table, i.e. for each interval of the discretization. The algorithm will cope with this constraint.

The Khiops method is based on a greedy bottom-up algorithm. It starts with initial single value intervals and then searches for the best merge between adjacent intervals. Two different types of merges are encountered. First, merges with at least one interval that does not meet the constraint and second, merges with both intervals fulfilling the constraint. The best merge candidate (with the highest chi-square value) is chosen in priority among the first type of merges (in which case the merge is accepted unconditionally), and otherwise, if all minimum frequency constraints are respected, among the second type of merges (in which case the merge is accepted under the condition of improvement of the confidence level). The algorithm is reiterated until both all minimum frequency constraints are respected and no further merge can decrease the confidence level.

Algorithm. Khiops

1. Initialization

1.1. Sort the explanatory attribute values

1.2. Create an elementary interval for each value

2. Optimization of the discretization

Repeat the following steps:

2.1. Search for the best merge

Search among the merges with at least one interval that does not meet the frequency constraint if anyone exists, among any merge otherwise

Merge that maximizes the chi-square value

2.2. Evaluate the stopping rule

Stop if all constraints are respected and if no further merge decreases the confidence level

2.3. Merge and continue if the stopping rule is not met

\subsection{Minimum frequency per interval}

In order to be reliable, the chi-square test requires that every cell of the contingency table has an expected value of at least 5 . This reliability constraint is equivalent to a minimum frequency constraint for each interval of the discretization.

The purpose of the Khiops discretization algorithm is to approximate the true class attribute distribution from the observed distribution of the training sample on the basis of intervals, which result from a supervised merging process. This process can be considered as an inductive algorithm, therefore subject to overfitting. In order to prevent overfitting, a solution is to increase the minimum frequency per interval constraint. The Khiops algorithm uses a heuristic control of overfitting by constraining the intervals to have a frequency greater than the square root of the sample size. This value allows both to improve the 
statistical reliability of the observed distribution in each interval and to refine the precision of the discretization owing to a potentially higher number of intervals when the sample size increases.

To improve the chi-square test reliability and to prevent overfitting, the Khiops algorithm is constrained to employ the maximum of the two preceding values.

\subsection{Computational complexity}

The computational complexity of the Khiops discretization method is evaluated on the basis of a sample of size $N$. With a straightforward implementation of the Khiops algorithm, the computational complexity is $O\left(N^{3}\right)$ :

- Initialization: $O(N \log (N))$

- Repeat (at most $N$ steps)

- Evaluate all possible merges between adjacent intervals: $N$ evaluations of the chi-square value (each evaluation requires $N$ steps)

- Search for the best merge: $O(N)$

- Evaluate the stopping rule: $O(1)$

We will show that the algorithm can be optimized, so that its computational complexity can be decreased to $O(N \log (N))$.

The chi-square value is additive and can be decomposed on the rows of the contingency table. The computation of the chi-square value on a whole contingency table requires $N$ computation steps to evaluate the contributions of rows.

$$
\text { Chi } 2=\sum_{i} \text { Chi } 2 r_{i}
$$

Computation of the chi-square value corresponding to the merge of two rows (i.e. intervals) $i$ and $i^{\prime}\left(i^{\prime}=i+1\right)$ can be formulated in the following way:

$$
\begin{aligned}
& \operatorname{Chi}^{\prime} M_{i i^{\prime}}=\sum_{k<i} \operatorname{Chi}^{2} r_{k}+\text { Chi2 }_{i i^{\prime}}+\sum_{k>i^{\prime}} \operatorname{Chi} 2 r_{k}
\end{aligned}
$$

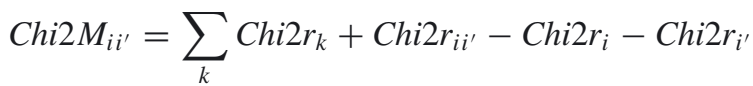

$$
\begin{aligned}
& \text { Chi2 } M_{i i^{\prime}}=\text { Chi } 2+\text { DeltaChi2 }_{i i^{\prime}}
\end{aligned}
$$

Owing to the additivity of the chi-square criterion, the chi-square value resulting from the merge between two adjacent intervals can be evaluated in a single step, if the initial chi-square value is available. If all the chi-square row values and DeltaChi2 values are kept in memory, then the search for the best merge is equivalent to the search of the best DeltaChi2. After a merge is completed, the chi-square row values and DeltaChi2 values need to be updated only for the new interval and its adjacent intervals to prepare the next merge step. 
The critical part of the algorithm corresponds to the search for the best merge. This search needs $N$ steps. If the list of all possible merges is initially sorted, and if this list remains sorted during the discretization process, the search for the best merge takes one step, at the expense of the cost needed to keep the list sorted. Balanced binary search trees (such as AVL binary search trees for example) allow to keep a list sorted when elements are inserted or deleted, with a logarithmic computational complexity.

Finally, the computational complexity of the optimized version of Khiops algorithm can be decreased to $O(N \log (N))$ if the chi-square row values and the DeltaChi2 values are kept in memory, if the chi-square values are computed in an additive way and if the merges are stored in a maintained sorted list.

The memory requirement of the algorithm is also $O(N \log (N))$. Data that need to be kept in memory are the $N$ chi-square row values, the $N$ DeltaChi2 values and the sorted list of merges, which has a memory requirement of $O(N \log (N))$.

Algorithm. Optimized Khiops

\section{Initialization}

1.1. Sort the explanatory attribute values: $O(N \log (N))$

1.2. Create an elementary interval for each value: $O(N)$

1.3. Compute the chi-square row values and the initial chi-square value: $O(N)$

1.4. Compute the DeltaChi2 values: $O(N)$

1.5. Sort the possible merges: $O(N \log (N))$

1.6. Compute the confidence level of this first discretization: $O(1)$

2. Optimization of the discretization

Repeat the following steps: at most $N$ steps

2.1. Search for the best possible merge: $O(1)$

2.2. Evaluate the stopping rule: $O(1)$

Stop if all constraints are respected and if no further merge decreases the confidence level

2.3. Merge and continue if the stopping rule is not met

2.3.1. Compute the chi-square row value for the new interval: $O(1)$

2.3.2. Compute the DeltaChi2 values for the two intervals adjacent to the new interval: $O(1)$

2.3.3. Update the sorted list of merges: $O(\log (N))$

Remove the merge just completed

Remove the two merges of the intervals adjacent to the former sub-intervals of the new interval

Add the two merges of the intervals adjacent to the new interval

The optimized version of the Khiops algorithm has the same computational complexity as the optimized version of the ChiMerge algorithm. This property allows the method to be effective on large databases (containing at least $10^{5}$ instances). 


\subsection{Practical issues}

There is a potential gap between the principles and the implementation of the algorithm. The principle of the algorithm is to search, among all possible sets of intervals, the set that minimizes the confidence level of the test of independence between the discretized attribute and the class attribute. This confidence level is evaluated with the chi-square statistic applied to the corresponding contingency table. To improve the statistical reliability of the algorithm, a minimum frequency related to the sample size is used to constrain the search of the best set of intervals. Based on these principles, the Khiops method seems robust. However, problems so far overlooked are now discussed.

The value used for the minimum frequency constraint is a heuristic choice and has no strong foundation. To be more consistent, this value should result from some statistical estimation that precisely takes into account the distribution of the class values and controls the probability of overfitting. Such an estimation, involving complex calculation, is beyond the scope of this paper.

The search algorithm is a greedy algorithm that tries to follow the minimum frequency constraint in a very simple and flexible way. This heuristic leads to super-linear computation time, which is mandatory as soon as large databases are processed. However, the search algorithm can be trapped in a local optimum and has no guarantee to find the best set of intervals. It is still not realistic to find the best discretization when computation time is an issue.

The algorithm needs a good evaluation of the chi-square statistic for very large chi-square values and degrees of freedom. Such a good evaluation is not presently available in standard numerical libraries. Furthermore, the limits of numerical precision of computer are rapidly overtaken when the confidence level gets too close to zero.

The practical limits of the Khiops discretization method are principally related to its implementation. The most critical issue is the evaluation of the chi-square statistic in a very large numerical domain. This problem has been studied and solved in Boullé (2001). The solution relies on a good approximation of the logarithm of the confidence level, and for better accuracy, on a precise evaluation of the variation threshold of the chi-square value that controls the stopping rule used in the Khiops algorithm.

We illustrate that in extensive experiments, in spite of the raw value used for the minimum frequency constraint and of the greedy search algorithm, the Khiops algorithm allows obtaining high quality discretizations together with fast computation time.

\section{Theoretical comparison with the chi-square-based discretization methods}

In this section, we compare the Khiops method with the related ChiMerge and ChiSplit methods, and show that the Khiops method solves several weaknesses of the other methods.

\subsection{Properties of merges in the Khiops method}

In this section, we demonstrate that when two rows of a contingency table are merged, the whole chi-square value can only decrease. However, after a merge, the chi-square statistic 
is based on fewer degrees of freedom. If the whole chi-square value decreases very little (or does not decrease at all), the related confidence level also decreases; otherwise the confidence level increases.

Theorem. When two rows of a contingency table are merged, the chi-square value of the contingency table decreases.

Proof: Let $p_{1}, p_{2}, \ldots, p_{J}$ be the probabilities of the class values in the complete contingency table.

$$
\sum_{j} p_{j}=1
$$

Let $a_{1}, a_{2}, \ldots, a_{J}$ and $b_{1}, b_{2}, \ldots, b_{J}$ be the probabilities of the class values in two adjacent rows of the contingency table with row frequencies $n$ and $n^{\prime}$.

$$
\sum_{j} a_{j}=1 . \quad \sum_{j} b_{j}=1 .
$$

The observed and expected frequencies are $a_{j} n$ and $p_{j} n$ in the first row, $b_{j} n^{\prime}$ and $p_{j} n^{\prime}$ in the second row. The chi-square row values Chi2r and Chi2r' are

$$
\text { Chi } 2 r=n\left(\sum_{j} a_{j}^{2} / p_{j}-1\right) \quad \text { and } \quad C h i 2 r^{\prime}=n^{\prime}\left(\sum_{j} b_{j}^{2} / p_{j}-1\right)
$$

Let us consider the merge of the two rows. The observed and expected frequencies in the merged row are $a_{j} n+b_{j} n^{\prime}$ and $p_{j}\left(n+n^{\prime}\right)$.

The chi-square merged row value $C h i 2 l^{\prime \prime}$ is

$$
C h i 2 r^{\prime \prime}=\left(n+n^{\prime}\right)\left(\sum_{j} \frac{\left(\left(a_{j} n+b_{j} n^{\prime}\right) /\left(n+n^{\prime}\right)\right)^{2}}{p_{j}}-1\right)
$$

The merge between the two rows causes an update of the whole chi-square value DeltaChi2 $=$ Chi $2 r^{\prime \prime}-$ Chi $2 r-$ Chi $2 r^{\prime}$.

$$
\begin{aligned}
& \text { DeltaChi } 2=\sum_{j} \frac{\left(n+n^{\prime}\right)\left(\left(a_{j} n+b_{j} n^{\prime}\right) /\left(n+n^{\prime}\right)\right)^{2}-n a_{j}^{2}-n^{\prime} b_{j}^{2}}{p_{j}} \\
& \text { DeltaChi } 2=-\frac{n n^{\prime}}{n+n^{\prime}} \sum_{j} \frac{\left(a_{j}-b_{j}\right)^{2}}{p_{j}}
\end{aligned}
$$

This last formula shows that the chi-square value of the contingency table decreases after the merge. 


\subsection{Comparison with the ChiMerge method}

For the ChiMerge discretization method, let us consider the local contingency table restricted to the two rows. Let $q_{1}, q_{2}, \ldots, q_{J}$. be the probabilities of the class values in this local contingency table.

$$
\sum_{j} q_{j}=1 . \quad q_{j}=\left(a_{j} n+b_{j} n^{\prime}\right) /\left(n+n^{\prime}\right) .
$$

In order to evaluate the merge of the two rows, let us calculate the local chi-square value.

$$
\begin{aligned}
& \text { LocalChi2 }=n\left(\sum_{j} a_{j}^{2} / q_{j}-1\right)+n^{\prime}\left(\sum_{j} b_{j}^{2} / q_{j}-1\right) \\
& \text { LocalChi2 }=\frac{n n^{\prime}}{n+n^{\prime}} \sum_{j} \frac{\left(a_{j}-b_{j}\right)^{2}}{q_{j}}
\end{aligned}
$$

The stopping rules used in the Khiops and ChiMerge methods are based on similar mathematical formulas, but the two discretization methods lead to a large difference in interpretation of the formulas. The probabilities of the class values are global to the whole contingency table for the Khiops method ( $p_{j}$ probabilities) whereas they are local to the two rows for the ChiMerge method ( $q_{j}$ probabilities).

For the Khiops method, the stopping rule is:

$$
\operatorname{Prob}(\text { Chi2 }+ \text { DeltaChi2, }(I-2) *(J-1))<\operatorname{Prob}(\text { Chi2 },(I-1) *(J-1))
$$

For the ChiMerge method (with a user parameter ProbThreshold), the stopping rule is:

$$
\operatorname{Prob}(\text { LocalChi2, J - 1) > ProbThreshold }
$$

This demonstrates an important difference between the two methods. The ChiMerge method acts locally, whereas the Khiops method takes into account the whole distribution of the class values, the whole number of intervals and the global chi-square value.

Example. We present an example in Table 2, which illustrates the difficulty in choosing the ProbThreshold user parameter in the ChiMerge algorithm. In Table 2, the initial contingency table on the left summarizes a sample with 1000 instances and two equidistributed class values. The rows (i.e intervals) of the initial table have an increasing proportion of the first class value, and the successive pairs of intervals have similar proportions of class values. The "natural" discretization of this table ends with the final contingency table shown on the right. The DeltaChi2 and LocalChi2 (with related confidence level) evaluations of the possible merges in the Khiops and ChiMerge methods are presented in the middle columns. The grayed values correspond to the preferred merges for each method. 
Table 2. Evaluation of the possible merges for the Khiops and ChiMerge methods.

\begin{tabular}{rrrrrrr}
\hline & Initial table & Khiops & \multicolumn{2}{c}{ ChiMerge } & \multicolumn{2}{c}{ Final table } \\
\hline 0 & 100 & $\Delta$ Chi2 & LChi2 & Prob & & \\
6 & 94 & -0.72 & 6.19 & 0.013 & 6 & 194 \\
24 & 76 & -6.48 & 12.71 & 0.000 & & \\
30 & 70 & -0.72 & 0.91 & 0.339 & 54 & 146 \\
47 & 53 & -5.78 & 6.10 & 0.013 & & \\
53 & 47 & -0.72 & 0.72 & 0.396 & 100 & 100 \\
70 & 30 & -5.78 & 6.10 & 0.013 & & \\
76 & 24 & -0.72 & 0.91 & 0.339 & 146 & 54 \\
94 & 6 & -6.48 & 12.71 & 0.000 & & \\
100 & 0 & -0.72 & 6.19 & 0.013 & 194 & 6 \\
\hline
\end{tabular}

The initial contingency table chi-square value is 449.2. Based on formula 6, we can derive the threshold of the DeltaChi2 value for the stopping rule, for given degrees of freedom and chi-square value. The DeltaChi2 table given in Boullé (2001) indicates that in the case of the present example, the merges are accepted when the value of DeltaChi2 is greater than -5 . With the Khiops algorithm, the five "obvious" merges are accepted and considered as equivalent. Using the ChiMerge algorithm, the central merges (near $p=0.5$ ) are fundamentally favored compared to the border merges (near $p=0$ and $p=1$ ). The "curious" merge (30-70) + (47-53) is even preferred to the "obvious" merge $(0-100)+$ (6-94). It is thus difficult to choose a good ProbThreshold for the ChiMerge stopping rule.

This example shows that the ChiMerge method has several intrinsic weaknesses that are solved by the Khiops method. The ChiMerge method is based on strictly local evaluation of the discretization. This makes the stopping rule parameter very difficult to tune. The local evaluation is incorrectly biased in favor of intervals with balanced distribution of the class values. Depending on the choice of the probability threshold, the merge process may stop either to soon or too late to detect the interesting intervals. The Khiops algorithm solves these problems by the use of a global evaluation of the discretization and of an automatic adaptable stopping rule criterion that allows a fair estimation of each merge and of its impact on the whole discretization.

\subsection{Comparison with the ChiSplit method}

Since the Khiops method is a bottom-up algorithm and the ChiSplit method is a top-down algorithm, the comparison is harder than for the ChiMerge method. The stopping rule used in the ChiSplit algorithm is difficult to tune because it relies on scaling factors (sample size), on the strength of the specific intervals to extract and on the position of these intervals in the contingency table.

Example. The same example described for the ChiMerge comparison illustrates the comparison with the ChiSplit method in Table 3. The global chi-square value of the split and its associated confidence level are presented in the ChiSplit columns. 
Table 3. Evaluation of the possible merges for the Khiops and ChiSplit methods.

\begin{tabular}{rrrrccc}
\hline Initial table & Khiops & \multicolumn{2}{c}{ ChiSplit } & \multicolumn{2}{c}{ Final table } \\
\hline 0 & 100 & $\Delta$ Chi2 & Chi2S & Prob & & \\
6 & 94 & -0.72 & 111.11 & $5.59 \mathrm{E}-26$ & 6 & 194 \\
24 & 76 & -6.48 & 220.90 & $5.76 \mathrm{E}-50$ & & \\
30 & 70 & -0.72 & 274.29 & $1.32 \mathrm{E}-61$ & 54 & 146 \\
47 & 53 & -5.78 & 326.67 & $5.11 \mathrm{E}-73$ & & \\
53 & 47 & -0.72 & 327.18 & $3.95 \mathrm{E}-73$ & 100 & 100 \\
70 & 30 & -5.78 & 326.67 & $5.11 \mathrm{E}-73$ & & \\
76 & 24 & -0.72 & 274.29 & $1.32 \mathrm{E}-61$ & 146 & 54 \\
94 & 6 & -6.48 & 220.90 & $5.76 \mathrm{E}-50$ & & \\
100 & 0 & -0.72 & 111.11 & $5.59 \mathrm{E}-26$ & 194 & 6 \\
\hline
\end{tabular}

According to Table 3, the confidence level used for the ChiSplit stopping rule should be set between $10^{-25}$ and $10^{-75}$. For larger sample sizes (more than 10000 instances), this confidence level would be above the limit of numerical precision of computers (about $10^{-300}$ ), and thus the choice of a confidence level becomes impossible. Furthermore, the best split found by the ChiSplit method lies exactly in the middle of the contingency table. This split produces two intervals (107-393) and (393-107) and is actually an excellent split of the table into two intervals. However, this split has definitely separated rows (47-53) and (53-47) that should be "intuitively" merged together.

Problem of nested interesting intervals. The ChiSplit top-down approach has a more serious drawback. It cannot discover interesting intervals nested between regular intervals since this requires two successive splits with the first one not very significant. We demonstrate this rigorously with the use of artificial data, consisting of two equidistributed intervals I1 and I3 with global frequency 1000, surrounding one interesting interval I2 with frequency 50:

- I1: (250-250)

- I2: (50-0)

- I3: (250-250)

With the ChiSplit method, the two possible splits have a chi-square value of 2.17 related to a confidence level of 0.14. With a ChiSplit stopping criterion threshold set to 0.05, the two splits are rejected and thus the interesting interval cannot be found. With the Khiops method, the initial contingency table has a chi-square value of 47.7 related to a confidence level of $4.310^{-11}$. The two possible merges are rejected because they increase the confidence level, and the interesting interval is therefore correctly identified.

In figure 1, we study the impact of the position of the interesting interval I2 (i.e. the number of instances in I1) on the behavior of the methods. Table A stands for the bipartition $\{\mathrm{I} 1 \cup \mathrm{I} 2, \mathrm{I} 3\}$ of the initial intervals. Table A can be seen both as a split after the interesting interval for the ChiSplit method and as a merge of the two first intervals for the Khiops 


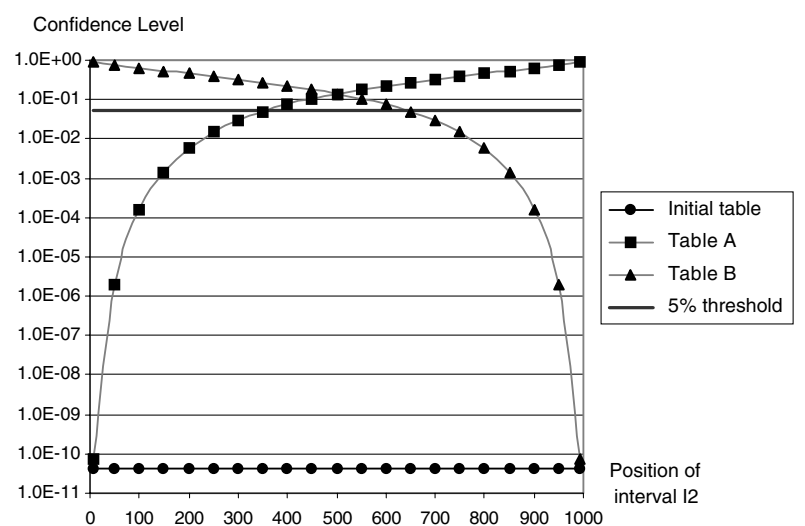

Initial table

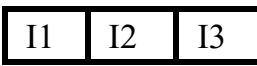

Table A

\begin{tabular}{|ll|l|}
\hline I1 & I2 & I3 \\
\hline
\end{tabular}

Table B

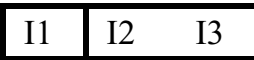

Figure 1. Confidence level related to the chi-square test for the initial table $\{\mathrm{I} 1, \mathrm{I} 2, \mathrm{I} 3\}$, the Table $\mathrm{A}\{\mathrm{I} 1 \cup \mathrm{I} 2, \mathrm{I} 3\}$ and the Table B $\{\mathrm{I} 1, \mathrm{I} 2 \cup \mathrm{I} 3\}$.

method. Table $B$ is defined in the same way with a partition $\{\mathrm{I} 1, \mathrm{I} 2 \cup \mathrm{I} 3\}$ of the initial intervals. Figure 1 shows that with the Khiops method, the confidence level of the initial table is always below that of the two tables resulting from the merges. The merges are thus rejected and the Khiops method always correctly detects the interesting interval. With the ChiSplit method, the best split among Table A and Table B corresponds to the table with the lowest confidence level. The ChiSplit method therefore detects the interesting interval only if it is situated at the beginning of the contingency table (before position 350 and the split leads to Table A) or at the end (after position 650 and the split leads to Table B). However, in about one third of the positions, when the interesting interval is around the middle of the contingency table, both splits are rejected because their confidence level are above the 5\% threshold stopping criterion of the algorithm.

The principle used in the ChiSplit method that combines a top-down approach and a greedy algorithm exhibits thus several weaknesses that may prevent the discovery of local patterns in discretized attributes.

\section{Experiments}

In our experimental study, we compare the Khiops method with other supervised and unsupervised discretization algorithms considered as a preprocessing step of the Naive Bayes classifier. The Naive Bayes classifier (Langley, Iba, \& Thompson, 1992) assigns the most probable class value given the explanatory attribute values, assuming independence between the attributes for each class value. After the discretization preprocessing step, the probabilities of continuous attributes are estimated using counts in each interval.

We gathered 15 datasets from U.C. Irvine repository (Blake, 1998), each dataset has at least one continuous attribute and at least a few tens of instances for each class value in order to perform reliable tenfold cross-validations. Table 4 describes the datasets; the last column corresponds to the relative frequency of the majority class. 


\begin{tabular}{|c|c|c|c|c|c|}
\hline Dataset & $\begin{array}{l}\text { Continuous } \\
\text { attributes }\end{array}$ & $\begin{array}{l}\text { Nominal } \\
\text { attributes }\end{array}$ & Size & Class values & Majority class \\
\hline Adult & 7 & 8 & 48842 & 2 & 76.07 \\
\hline Australian & 6 & 8 & 690 & 2 & 55.51 \\
\hline Breast & 10 & 0 & 699 & 2 & 65.52 \\
\hline Crx & 6 & 9 & 690 & 2 & 55.51 \\
\hline German & 24 & 0 & 1000 & 2 & 70.00 \\
\hline Heart & 10 & 3 & 270 & 2 & 55.56 \\
\hline Hepatitis & 6 & 13 & 155 & 2 & 79.35 \\
\hline Hypothyroid & 7 & 18 & 3163 & 2 & 95.23 \\
\hline Ionosphere & 34 & 0 & 351 & 2 & 64.10 \\
\hline Iris & 4 & 0 & 150 & 3 & 33.33 \\
\hline Pima & 8 & 0 & 768 & 2 & 65.10 \\
\hline SickEuthyroid & 7 & 18 & 3163 & 2 & 90.74 \\
\hline Vehicle & 18 & 0 & 846 & 4 & 25.77 \\
\hline Waveform & 21 & 0 & 5000 & 3 & 33.92 \\
\hline Wine & 13 & 0 & 178 & 3 & 39.89 \\
\hline
\end{tabular}

The discretization methods studied in the comparison are:

- Khiops: the method described in this paper

- MDLPC: Minimum Description Length Principal Cut (Fayyad, 1992)

- ChiMerge: bottom-up method based on chi-square (Kerber, 1991)

- ChiSplit: top-down method based on chi-square (Bertier \& Bouroche, 1981)

- Equal Width

- Equal Frequency

The Khiops and MDLPC methods have an automatic stopping rule and do not require any parameter setting. For the ChiMerge and ChiSplit methods, the significance level is set to 0.95 for chi-square threshold. For the Equal Width and Equal Frequency unsupervised discretization methods, the number of intervals is set to 10 . We have re-implemented these alternative discretization approaches in order to eliminate any variance resulting from different cross-validation splits.

As the purpose of our experimental study is to compare the discretization methods, we chose to ignore nominal attributes to build the Naive Bayes classifiers. We ran a stratified tenfold cross-validation and report the mean and the standard deviation of the accuracies. In order to determine whether accuracies are significantly different between the Khiops method and the alternative methods, the $t$-statistic of the difference of the accuracies is computed. Under the null hypothesis, this value has a Student's distribution with 9 degrees of freedom. A two-tailed test is appropriate because we do not know in advance whether 
Table 5. Accuracies of the Naive Bayes classifier with different discretization methods.

\begin{tabular}{|c|c|c|c|c|c|c|}
\hline Dataset & Khiops & MDLPC & ChiMerge & ChiSplit & Eq. width & Eq. freq. \\
\hline Adult & $83.1 \pm 0.5$ & $84.4 \pm 0.5-$ & $77.8 \pm 0.7+$ & $84.3 \pm 0.5-$ & $81.2 \pm 0.4+$ & $81.1 \pm 0.6+$ \\
\hline Australian & $78.1 \pm 3.9$ & $77.4 \pm 3.6$ & $75.1 \pm 4.6$ & $78.1 \pm 3.5$ & $71.0 \pm 5.3+$ & $80.4 \pm 2.4-$ \\
\hline Breast & $97.3 \pm 1.2$ & $97.1 \pm 1.1$ & $90.1 \pm 3.5+$ & $97.0 \pm 1.7$ & $96.6 \pm 1.7+$ & $97.4 \pm 1.4$ \\
\hline Crx & $77.2 \pm 4.9$ & $76.5 \pm 5.8$ & $71.4 \pm 5.9+$ & $78.0 \pm 7.0$ & $70.3 \pm 3.6+$ & $79.7 \pm 6.1-$ \\
\hline German & $75.5 \pm 3.4$ & $72.5 \pm 1.8+$ & $74.3 \pm 3.6$ & $75.6 \pm 4.2$ & $75.5 \pm 3.6$ & $75.5 \pm 3.9$ \\
\hline Heart & $78.1 \pm 7.5$ & $80.7 \pm 8.6$ & $72.2 \pm 6.3+$ & $78.9 \pm 8.8$ & $81.1 \pm 6.5-$ & $80.7 \pm 6.6-$ \\
\hline Hepatitis & $78.8 \pm 12$ & $76.8 \pm 13$ & $81.5 \pm 11$ & $78.9 \pm 9.5$ & $82.6 \pm 8.5$ & $78.8 \pm 11$ \\
\hline Hypothyroid & $98.0 \pm 1.1$ & $98.7 \pm 0.6-$ & $98.2 \pm 0.6$ & $98.5 \pm 1.0$ & $97.4 \pm 0.6$ & $97.6 \pm 0.9+$ \\
\hline Ionosphere & $89.7 \pm 3.2$ & $90.9 \pm 4.4$ & $86.1 \pm 5.2$ & $86.3 \pm 4.0$ & $89.5 \pm 4.4$ & $91.2 \pm 3.9$ \\
\hline Iris & $92.0 \pm 2.7$ & $92.7 \pm 2.0$ & $94.7 \pm 2.7-$ & $94.0 \pm 3.6$ & $95.3 \pm 4.3$ & $94.7 \pm 5.0$ \\
\hline Pima & $75.1 \pm 4.4$ & $76.2 \pm 2.1$ & $72.0 \pm 2.7$ & $75.0 \pm 3.0$ & $74.7 \pm 3.1$ & $74.0 \pm 3.5$ \\
\hline SickEuthyroid & $96.3 \pm 1.0$ & $95.9 \pm 1.1$ & $96.3 \pm 1.3$ & $95.8 \pm 1.0$ & $92.9 \pm 1.8+$ & $93.2 \pm 1.1+$ \\
\hline Vehicle & $61.5 \pm 2.9$ & $60.2 \pm 2.3$ & $64.5 \pm 3.8-$ & $63.1 \pm 4.1$ & $63.6 \pm 3.3$ & $61.4 \pm 3.3$ \\
\hline Waveform & $81.0 \pm 1.0$ & $80.8 \pm 0.8$ & $75.9 \pm 1.6 \mathrm{~g}+$ & $80.0 \pm 1.8+$ & $80.8 \pm 1.2$ & $80.7 \pm 1.2$ \\
\hline Wine & $96.7 \pm 2.7$ & $96.7 \pm 3.7$ & $96.6 \pm 4.5$ & $95.0 \pm 3.9$ & $95.5 \pm 6.5$ & $96.6 \pm 3.7$ \\
\hline Mean & 83.9 & 83.8 & 81.8 & 83.9 & 83.2 & 84.2 \\
\hline+ number & & 1 & 5 & 1 & 5 & 3 \\
\hline - number & & 2 & 2 & 1 & 1 & 3 \\
\hline
\end{tabular}

the mean of the Khiops accuracies is likely to be greater than that of the alternative method or vice versa. The confidence level is set to $5 \%$.

Table 5 shows the mean and the standard deviation of the accuracies of the Naive Bayes induction algorithm. The significant wins for the Khiops method are indicated with + , and the significant losses with -. The Khiops, MDLPC, ChiSplit and EqualFrequency methods have similar results, and perform better than the ChiMerge and EqualWidth methods. These results allow to distinguish two groups of methods, but are not conclusive enough to further rank the methods. The case of the EqualFrequency method ( 3 wins and 3 losses for the Khiops method) shows that there is a need for more experiments.

In order to analyze the performance of the discretization algorithms more fully, we proceed with the same experiment for each individual continuous attribute in every dataset. These additional experiments are equivalent to 181 experiments with single-attribute datasets. Each discretization method can be evaluated as an elementary attribute classifier that predicts the more frequent class value in each learned interval. The results are summarized in Table 6, which reports for each dataset the mean of the dataset attribute accuracies and the number of significant wins and losses of the elementary attribute classifiers when compared with the Khiops method.

This experiment is more informative than the previous one. It allows a better comparison between the discretization methods, eliminating the bias of the choice of a specific induction 
Table 6. Means of accuracies, number of significant wins and losses per dataset, for the elementary attribute classifiers.

\begin{tabular}{|c|c|c|c|c|c|c|c|c|c|c|c|c|c|c|c|c|}
\hline \multirow[b]{2}{*}{ Dataset } & \multirow[b]{2}{*}{ Khiops } & \multicolumn{3}{|c|}{ MDLPC } & \multicolumn{3}{|c|}{ ChiMerge } & \multicolumn{3}{|c|}{ ChiSplit } & \multicolumn{3}{|c|}{ Eq. width } & \multicolumn{3}{|c|}{ Eq. freq. } \\
\hline & & & + & - & & + & - & & + & - & & + & - & & + & - \\
\hline Adult & 77.2 & 77.3 & & 2 & 75.7 & 2 & 2 & 77.3 & 1 & 2 & 76.8 & 2 & & 76.6 & 2 & \\
\hline Australian & 64.5 & 65.0 & & 1 & 64.7 & & & 65.1 & & 1 & 61.4 & 3 & 1 & 65.7 & & \\
\hline Breast & 86.0 & 86.1 & 1 & 1 & 85.6 & & & 85.9 & & & 86.0 & & & 85.7 & 1 & \\
\hline Crx & 64.5 & 65.2 & & & 63.8 & 1 & & 65.3 & & & 61.1 & 3 & & 65.6 & & \\
\hline German & 70.0 & 70.0 & & & 70.0 & & & 70.1 & & & 70.1 & & 1 & 70.0 & & \\
\hline Heart & 63.8 & 64.0 & & & 64.0 & & & 63.8 & & & 63.9 & & & 64.5 & & \\
\hline Hepatitis & 79.4 & 79.3 & & & 77.8 & 3 & & 79.3 & & & 79.8 & & & 79.9 & & \\
\hline Hypothyroid & 96.0 & 96.1 & & & 96.0 & 3 & & 96.1 & 1 & & 95.4 & 3 & & 95.2 & 3 & \\
\hline Ionosphere & 78.7 & 77.6 & 6 & 5 & 75.7 & 14 & & 79.5 & & 7 & 73.9 & 14 & 1 & 75.0 & 17 & \\
\hline Iris & 77.7 & 75.5 & 1 & & 77.0 & & & 78.8 & & & 76.5 & 1 & & 76.3 & & \\
\hline Pima & 66.8 & 66.1 & 2 & & 65.6 & 2 & & 66.5 & & & 66.8 & & & 66.3 & & 1 \\
\hline SickEuthyroid & 91.4 & 91.3 & & & 91.3 & 1 & & 91.3 & & & 90.7 & 2 & & 91.0 & 1 & \\
\hline Vehicle & 40.9 & 40.5 & 3 & 2 & 41.4 & 1 & 4 & 42.1 & & 4 & 40.8 & 1 & 1 & 40.3 & 2 & \\
\hline Waveform & 49.1 & 49.37 & & & 48.7 & 5 & & 49.1 & 1 & 1 & 49.2 & 3 & 1 & 49.5 & 1 & 3 \\
\hline \multirow[t]{2}{*}{ Wine } & 62.0 & 60.1 & 2 & & 59.6 & 2 & & 60.4 & 1 & & 61.4 & 4 & 1 & 60.8 & 1 & \\
\hline & 68.4 & 68.0 & 15 & 11 & 67.4 & 34 & 6 & 68.6 & 4 & 15 & 67.2 & 36 & 6 & 67.6 & 28 & 4 \\
\hline
\end{tabular}

algorithm. The results show that supervised methods (except ChiMerge) perform clearly better than unsupervised methods. The ChiMerge method is slightly better than the EqualWidth method, but not as good as the EqualFrequency method. The Khiops method and the MDLPC method are clearly better than the EqualFrequency, ChiMerge and EqualWidth methods, with a slight advantage in favor of the Khiops method over the MDPLC method. The ChiSplit method obtains the best results of the experiments. A close look at Table 6 indicates a special behaviour of the ionosphere dataset, where the ChiSplit method largely dominates the other methods with 7 wins in the comparison with the Khiops method. An inspection of the discretizations performed by the ChiSplit algorithm reveals intervals with very small frequencies, that cannot be found by the Khiops algorithm because of its strict constraint of minimum frequency per interval.

Although both the Khiops method and the MDLPC method yield comparable results on average, they often differ on individual cases. For 181 discretized attributes, there are 15 wins for the Khiops method and 11 for the MDPLC method. It can be noticed that these two methods are based on very different approaches. The Khiops method is a bottom-up algorithm with a global criterion based on the chi-square statistic, whereas the MDLPC method is a top-down algorithm with a local criterion based on Shannon entropy.

Despite some theoretical weaknesses, the ChiSplit method obtains better results than the Khiops method. The analysis of partitions of intervals built by the Khiops method reveals some limitation related to the minimum frequency constraint. While this constraint clearly enhances the reliability of the Khiops method, it prevents it from discovering finely-grained 
patterns in numerical domains. In our future work, we plan to investigate on this minimum frequency constraint in order to improve the performance of the Khiops method.

The second set of experiments based on single attributes brings useful additional information to the first set of experiments based on the Naive Bayes classifier. All these results are based on 15 UCI datasets and 181 continuous attributes, and should be interpreted carefully. However, the main trends expressed by the results allow to rank the tested discretization methods in the following way:

1. ChiSplit

2. Khiops, MDLPC

3. EqualFrequency

4. ChiMerge, EqualWidth

The ChiSplit method is the best of the tested methods, while the Khiops method is at least as good as the MDPLC method.

\section{Conclusion}

The principle of the Khiops discretization method is to minimize the confidence level related to the test of independence between the discretized explanatory attribute and the class attribute. This optimization is based on the chi-square criterion applied to the whole set of intervals of the discretization. This global evaluation carries some intrinsic benefits compared with the connected ChiMerge and ChiSplit methods. The Khiops automatic stopping rule brings both ease of use and high quality discretizations. Its computational complexity is the same as for the fastest other discretization methods.

Extensive evaluations indicate notable accuracy results for the Khiops method. For further comparisons, we plan in future works to study other aspects of evaluation such as the robustness or the size of discretizations.

\section{Acknowledgments}

I am grateful to Fabrice Clérot and Jean-Emmanuel Viallet for many insightful discussions and careful proof reading of the manuscript. I also wish to thank the editor Prof. Peter Flach and the three anonymous reviewers for their beneficial comments.

\section{Note}

1. French patent No. 0107006.

\section{References}

Bertelsen, R., \& Martinez, T. R. (1994). Extending ID3 through discretization of continuous inputs. In Proceedings of the 7th Florida Artificial Intelligence Research Symposium (pp. 122-125). Florida AI Research Society.

Bertier, P., \& Bouroche, J. M. (1981). Analyse des données multidimensionnelles. Presses Universitaires de France. 
Blake, C. L., \& Merz, C. J. (1998). UCI Repository of machine learning databases [http://www.ics.uci.edu/ mlearn/MLRepository.html]. Irvine, CA: University of California, Department of Information and Computer Science.

Boullé, M. (2001). Khiops: Discrétisation des attributs numériques pour le Data Mining. Note technique NT/FTR\&D/7339. France Telecom R\&D.

Breiman, L., Friedman, J. H., Olshen, R. A., \& Stone, C. J. (1984). Classification and Regression Trees. California: Wadsworth International.

Burdsall, B., \& Giraud-Carrier, C. (1997). Evolving fuzzy prototypes for efficient data clustering. In Proceedings of the Second International ICSC Symposium on Fuzzy Logic and Applications (ISFL'97) (pp. 217-223).

Catlett, J. (1991). On changing continuous attributes into ordered discrete attributes. In Proceedings of the European Working Session on Learning (pp. 87-102). Springer-Verlag.

Dougherty, J., Kohavi, R., \& Sahami, M. (1995). Supervised and unsupervised discretization of continuous features. In Proceedings of the 12th International Conference on Machine Learning (pp. 194-202). San Francisco, CA: Morgan Kaufmann.

Fayyad, U., \& Irani, K. (1992). On the handling of continuous-valued attributes in decision tree generation. Machine Learning, 8, 87-102.

Holte, R. C. (1993). Very simple classification rules perform well on most commonly used datasets. Machine Learning, 11, 63-90.

Kass, G. V. (1980). An exploratory technique for investigating large quantities of categorical data. Applied Statistics, 29:2, 119-127.

Kerber, R. (1991). Chimerge discretization of numeric attributes. In Proceedings of the 10th International Conference on Artificial Intelligence (pp. 123-128).

Langley, P., Iba, W., \& Thompson, K. (1992). An analysis of bayesian classifiers. In Proceedings of the 10th National Conference on Artificial Intelligence (pp. 223-228). AAAI Press.

Quinlan, J. R. (1993). C4.5: Programs for machine learning. Morgan Kaufmann.

Zighed, D. A., Rabaseda, S., \& Rakotomalala, R. (1998). Fusinter: A method for discretization of continuous attributes for supervised learning. International Journal of Uncertainty, Fuzziness and Knowledge-Based Systems, $6: 33,307-326$.

Zighed, D. A., \& Rakotomalala, R. (2000). Graphes d'induction (pp. 327-359). HERMES Science Publications,

Received December 12, 2001

Revised June 10, 2003

Accepted June 11, 2003

Final manuscript August 2, 2003 